\title{
Evolution of International Psychiatry
}

\author{
Paolo Girardi ${ }^{1, *}$, Luca Bonanni ${ }^{2}\left(\mathbb{D}\right.$, Georgios D. Kotzalidis ${ }^{1}\left(\mathbb{D}\right.$, Federica Fiaschè ${ }^{2}$ \\ and Antonio Del Casale ${ }^{1}$ (D) \\ 1 Department of Dynamic and Clinical Psychology, Sapienza University, 00189 Rome, Italy; \\ giorgio.kotzalidis@uniroma1.it (G.D.K.); antonio.delcasale@uniroma1.it (A.D.C.) \\ 2 Faculty of Medicine and Psychology, Sapienza University, 00189 Rome, Italy; \\ luca.bonanni4@gmail.com (L.B.); fiaschefederica@libero.it (F.F.) \\ * Correspondence: paolo.girardi@uniroma1.it
}

Received: 31 August 2020; Accepted: 4 September 2020; Published: 14 September 2020

\begin{abstract}
International psychiatry is currently facing serious challenges triggered by the global economic crisis and the COVID-19 pandemic. These global events lead to the need to broaden our nosographic and therapeutic horizons, and to make use of the newest psychological approaches and the latest neuroscience acquisitions. The focus should be on the psychological consequences of the pandemic, not only on people suffering from mental disorders, but also on the general population, for which the risk of developing psychic symptoms appears to be increased. A population that needs special attention is that of health workers involved in the management of the pandemic. In facing these problems, psychiatry today can use numerous new clinical applications and technologies in the fields of precision medicine. These include genomics, neuroimaging, and microbiomics, which can also be integrated with each other through machine learning systems. They can provide new contributions both in treatment personalization and in the evolution of nosographic systems. Besides this, the contribution of psychotherapies and dynamic and clinical psychology appears to be indispensable for a complete understanding of the clinical and personological aspects of patients. This journal aims to include innovative studies deriving from original, clinical, and basic research in the fields of mental health, precision psychiatry, genomics, neuroimaging, neuropsychopharmacology, and dynamic and clinical psychology.
\end{abstract}

Keywords: psychiatry; precision medicine; COVID-19; neuroimaging; genomics; pharmacogenomics; microbiomics; machine learning; clinical psychology; dynamic psychology

\section{Introduction}

Psychiatry has an increasing need to make itself a global and international science, respecting the cultural peculiarities of the populations and considering contemporary problems. The serious issues induced by the COVID-19 pandemic and the global economic crisis have significant repercussions on human psychology, particularly in people with mental disorders. However, innovative techniques in precision medicine, including genomics, microbiomics, metabolomics, neuroimaging, and ground-breaking approaches such as machine learning and artificial intelligence, allow us to better address the problems related to the treatment of mental disorders.

\section{COVID-19 and Mental Health}

In a few months, COVID-19 has induced illness in millions of subjects, killing more than 850,000 people worldwide to date and disrupting the lives of virtually everyone. Psychiatry often addresses problems related to trauma and loss, but the experience of a global pandemic that directly threatens the life of most people is an extraordinary event. Current knowledge regarding the effects of pandemics on 
mental health comes from studies performed in previous outbreaks, which suggest high rates of acute stress and anxiety among ordinary people, patients, and healthcare professionals. Some health workers involved with emergency departments and intensive care units have experienced extreme stress. These same workers had to face the sense of frustration and impotence for the inevitable death of many patients and the constant concern for their own health and, most of all, the risk of transmission to loved ones and frail people of any infection contracted during work. Such experiences can have long-lasting emotional and functional consequences, as demonstrated by the high rates of depression and anxiety among emergency management health professionals [1]. There is increased stress in individuals with mental disorders and other people in social isolation, who have suddenly experienced reduced access to their usual treatment programs or support systems. Further studies are needed on the long-term consequences of this pandemic on individuals with mental disorders, who may have an increased risk of developing stress-related symptoms and/or suicidal ideation, but also on the general population. In fact, a rise in various stress-related disorders has been variably observed in the general population in various countries that often overcomes the clinical threshold [2].

New psychological syndromes have emerged in individuals who have begun to suffer from stress, anxiety, or pain because of the pandemic; some people have lost family members or significant others in traumatic circumstances, amidst a complete inability to take leave of their dying loved ones or offer them adequate burials. Another important issue concerns the physical distancing that can help prevent the further spread of the virus, but on the other hand can trigger psychological symptoms related to social isolation, especially in the elderly; the poor; and people with difficulties in handling technology, such as telephone or internet connections [2].

Another important aspect in today's scenario is economy. The spread of COVID-19 has caused a global economic crisis with inevitable repercussions on mental health. Economy and mental health are two interconnected areas that influence each other. This inevitably prompts governments to be guided by economic data in their decisions on the allocation of resources for mental health; we must be very alert to the risk of reducing the resources allocated to mental health. There is instead a need to increase these resources, merely by considering the current situation. Over the past 30 years, there has been a rapid growth in mental health economic planning. However, important gaps remain in different areas, including the treatment of perinatal depression, mental health in childhood and adolescence, the psychotherapeutic treatment of depression, community-based early intervention, and occupational support for patients with psychosis, as well as cognitive stimulation and other interventions for neurocognitive disorders, which represent the main challenges to focus upon in the future [3].

\section{Perspectives of Contemporary International Psychiatry}

International psychiatry has accumulated much new knowledge that could help to meet this new requirement. In recent years, research has increasingly focused on personalized medicine, with the aim to identify the most suitable therapies, in a certain sense "tailored" for each patient, based on the study of their genomics. Identifying the genes involved in the response to psychotropic drugs could be useful for improving therapeutic efficacy. Gene-drug interactions can affect drug safety, and pharmacogenomics could help identify the most suitable and tolerated drugs and dosages suited to each individual patient. Large prospective randomized studies are needed to quantify the value of genetic stratification of patients in neuropsychopharmacotherapy, so to be able to draw definitive conclusions on the clinical and economic benefits of preventive genotyping and, last but not least, to demonstrate a favorable cost-effectiveness profile of such interventions [4].

Another perspective is linked to machine learning models (or their development, deep learning), which aim to acquire statistical functions from multidimensional datasets to make generalizable predictions on individuals, with the pragmatic goal of translating research results to predict outcomes for single persons or groups of individuals. This approach holds promise with respect to the possibility to improve the accuracy of diagnoses, prognosis (outcome), and treatment for people with mental illness, starting from clinical and biological data. However, the initial promising results must 
not prompt an overly optimistic bias [5], since limitations could exist that restrict a wide clinical application of machine-learning data, just as they exist with any new emerging technology. Interestingly, deep/machine learning-data mining methods may be used for detecting and classifying Covid-19 disease [6], aiding clinical decisions [7], and forecasting the expansion of the infection [8].

Today, pharmacogenomics and machine learning are not isolated or abstract issues in psychiatry. A growing body of evidence suggests that precision psychiatry, an interdisciplinary field that includes psychiatry, precision medicine, and pharmacogenomics, forms an indispensable foundation for medical practices in general, and specifically psychiatric disorders. In the light of the latest advances in machine learning techniques and artificial intelligence, numerous biomarkers and genetic loci associated with psychiatric illness and relevant treatments are being identified in precision psychiatric research. There are new promising developments in this discipline, such as deep learning and neural network algorithms associated with multi-omics and neuroimaging data [9]. Innovative artificial intelligence and machine learning techniques could integrate precision psychiatry techniques to improve treatment prediction, prognosis, diagnosis, and the detection of potential biomarkers.

The use of neuroimaging has played a pivotal role for decades in the advancement of research in psychiatry and in the field of neuroscience. However, despite its considerable potential for mental health care, this approach still has few clinical applications, being largely confined to research. Contributions have been made to expanding its application, i.e., better understanding of psychopathology beyond current diagnostic boundaries, a personalized prediction regarding the response to treatment, improvements in the prognostic field, and the possibility of developing formal theories to configure new interventions [10].

A further important aspect concerns the study of the intestinal microbiota. In the past, the role of the brain-gut axis was not fully understood in mental health. However, currently the gut has been upgraded to a "virtual organ" that would produce molecules of primary importance for brain function and psychological wellbeing. Studies on the role of the microbiota in mental disorders, in particular, those related to stress and its disorders (depression, anxiety, and posttraumatic stress disorders), have made it possible to expand the range of therapeutic targets, not only for pharmacological interventions but also for nutritional and surgical (i.e., transplantation) ones. The absence of scientific insights in this area could prove to be one of the missing links that have limited significant therapeutic advances in psychiatry in recent decades [11]. It is interesting in this respect that Covid-19 infection alters the gut microbiome [12], and that beneficial intestinal microbes might be protective against Covid-19 [13].

Another facet of mental health that needs to be highlighted concerns the possibilities of further development in psychodynamic theories and psychotherapies. By emphasizing the formal diagnosis that seeks common characteristics in a group of individuals and ends up placing subjects that are often very different from each other in the same category, contemporary psychiatry risks losing the notion of "person", that is, what is absolutely unique and peculiar to each human being [14]. Preserving the person in the diagnostic and therapeutic process of psychiatry today implies a way of thinking that requires time and effort but does not reduce a person to a simple list of symptoms. Even if the current models of clinical practice are economy-oriented, hence making available increasingly shorter sessions and treatment durations, it is necessary to enlarge our views and humanize the entire field through emphasizing the doctor-patient encounter, wherein a correct framing of clinical reality may occur.

Author Contributions: P.G. and A.D.C. conceived the Editorial, A.D.C., L.B., F.F. and G.D.K. wrote the first draft, A.D.C., G.D.K., F.F., and L.B. performed literature searches, G.D.K., L.B. and F.F. wrote substantial portions of the text, P.G., A.D.C., and G.D.K. supervised the writing of the Editorial, F.F., L.B., A.D.C., P.G., and G.D.K. provided the final draft All authors have read and agreed to the published version of the manuscript.

Funding: This research received no external funding.

Conflicts of Interest: The authors declare no conflict of interest related to this article. 


\section{References}

1. Lai, J.; Ma, S.; Wang, Y.; Cai, Z.; Hu, J.; Wei, N.; Wu, J.; Du, H.; Chen, T.; Li, R.; et al. Factors Associated with Mental Health Outcomes Among Health Care Workers Exposed to Coronavirus Disease 2019. JAMA Netw. Open 2020, 3, e203976. [CrossRef] [PubMed]

2. Xiong, J.; Lipsitz, O.; Nasri, F.; Lui, L.M.; Gill, H.; Phan, L.; Chen-Li, D.; Iacobucci, M.; Ho, R.; Majeed, A.; et al. Impact of COVID-19 pandemic on mental health in the general population: A systematic review [published online ahead of print, 2020 Aug 8]. J. Affect. Disord. 2020, 277, 55-64. [CrossRef] [PubMed]

3. Knapp, M.; Wong, G. Economics and mental health: The current scenario. World Psychiatry 2020, 19, 3-14. [CrossRef]

4. Van Westrhenen, R.; Aitchison, K.J.; Ingelman-Sundberg, M.; Jukić, M.M. Pharmacogenomics of antidepressant and antipsychotic treatment: How far have we got and where are we going? Front. Psychiatry 2020, 11, 94. [CrossRef] [PubMed]

5. Dwyer, D.B.; Falkai, P.; Koutsouleris, N. Machine learning approaches for clinical psychology and psychiatry. Annu. Rev. Clin. Psychol. 2018, 14, 91-118. [CrossRef] [PubMed]

6. Khanday, A.M.U.D.; Rabani, S.T.; Khan, Q.R.; Rouf, N.; Mohi Ud Din, M. Machine learning based approaches for detecting COVID-19 using clinical text data. Int. J. Inf. Technol. 2020, 30, 1-9. [CrossRef] [PubMed]

7. Debnath, S.; Barnaby, D.P.; Coppa, K.; Makhnevich, A.; Kim, E.J.; Chatterjee, S.; Tóth, V.; Levy, T.J.; Paradis, M.D.; Cohen, S.L.; et al. Northwell COVID-19 Research Consortium. Machine learning to assist clinical decision-making during the COVID-19 pandemic. Bioelectron. Med. 2020, 6, 14. [CrossRef] [PubMed]

8. Albahri, A.S.; Hamid, R.A.; Alwan, J.K.; Al-Qays, Z.; Zaidan, A.A.; Zaidan, B.; Alamoodi, A.H.; Khlaf, J.M.; Almahdi, E.M.; Thabet, E.; et al. Role of biological Data Mining and Machine Learning techniques in detecting and diagnosing the novel Coronavirus (COVID-19): A systematic review. J. Med. Syst. 2020, 44, 122. [CrossRef] [PubMed]

9. Lin, E.; Lin, C.H.; Lane, H.Y. Precision psychiatry applications with pharmacogenomics: Artificial intelligence and machine learning approaches. Int. J. Mol. Sci. 2020, 21, 969. [CrossRef] [PubMed]

10. Zhang, X.; Braun, U.; Tost, H.; Bassett, D.S. Data-driven approaches to neuroimaging analysis to enhance psychiatric diagnosis and therapy. Biol. Psychiatry Cogn. Neurosci. Neuroimaging 2020, 5, 780-790. [CrossRef] [PubMed]

11. Dinan, T.G.; Cryan, J.F. Gut microbiota: A missing link in psychiatry. World Psychiatry 2020, 19, 111-112. [CrossRef] [PubMed]

12. Villapol, S. Gastrointestinal symptoms associated with COVID-19: Impact on the gut microbiome. Transl. Res. 2020, in press. [CrossRef] [PubMed]

13. Antunes, A.E.C.; Vinderola, G.; Xavier-Santos, D.; Sivieri, K. Potential contribution of beneficial microbes to face the COVID-19 pandemic. Food Res. Int. 2020, 136, 109577. [CrossRef] [PubMed]

14. Gabbard, G.O. Preserving the person in contemporary psychiatry. Psychiatr. Clin. N. Am. 2018, 41, $183-191$. [CrossRef] [PubMed]

(C) 2020 by the authors. Licensee MDPI, Basel, Switzerland. This article is an open access article distributed under the terms and conditions of the Creative Commons Attribution (CC BY) license (http://creativecommons.org/licenses/by/4.0/). 